\title{
Complications associated with percutaneous needle biopsy of the liver when one, two or three specimens are taken
}

\author{
Breminand Maharaj and Ishan G. Bhoora
}

\begin{abstract}
Department of Experimental and Clinical Pharmacology, University of Natal Medical School, PO Box 17039, Congella 4013, South Africa
\end{abstract}

\begin{abstract}
Summary: The diagnostic yield of blind percutaneous liver biopsy is improved when two or three specimens are taken during the procedure by redirecting the needle through a single entry-site, without exposing the patient to a greater risk of complications provided that standard precautions are taken. This study was designed to obtain further data on the safety of this procedure at King Edward VIII Hospital, Durban.

During the period 1984-1990 (inclusive) a total of 2,646 biopsies were carried out: a single specimen was obtained in 834 patients, two specimens in 983 patients and three in 829 patients. Complications directly attributable to the procedure occurred in 24 patients who had one specimen, 20 who had two, and 19 who had three specimens taken during the biopsy. A single specimen had been obtained from three of the eight patients who had died, two specimens had been taken from another patient, and three specimens were obtained from the other four patients, i.e. patients in whom two or three specimens were taken did not have a higher incidence of pain, symptomatic hypotension, biliary peritonitis or death than those in whom one specimen was taken. Accordingly, when blind percutaneous needle biopsy of the liver is carried out, two specimens should be obtained by redirecting the needle through a single entry site as this improves the diagnostic yield without increasing complications. The morbidity and mortality associated with liver biopsy in this hospital is, however, high. Good technique, careful monitoring of patients after biopsy and prompt and aggressive resuscitation are essential if the mortality rate is to be reduced.
\end{abstract}

\section{Introduction}

Liver biopsy, an important diagnostic procedure in patients in whom either diffuse or focal liver disease is suspected, is associated with complications, the major one being intra-peritoneal haemorrhage. When blind percutaneous needle biopsy of the liver is carried out, a single specimen is usually obtained. It has been shown that the diagnostic yield of liver biopsy is increased when two specimens are taken, and can be improved further by taking a third biopsy specimen during the procedure. ${ }^{1}$ It appeared that this could be achieved without exposing the patient to a greater risk of complications provided that standard precautions were taken. Since this study was carried out in a small group of patients, and since there was a paucity of data on the frequency and nature of complications that occur when two or more consecutive biopsy specimens are taken, a study was conducted to provide further data on the safety of this procedure.

Correspondence: Professor B. Maharaj, M.B., Ch.B., F.C.P.(S.A.), M.D.

Accepted: 27 May 1992

\section{Patients and methods}

This study was conducted in two parts. In the first part, the records of all patients in whom percutaneous liver biopsy had been carried out at King Edward VIII Hospital, Durban, South Africa during the period 1984-1987 (inclusive) were reviewed. The second part of the study was done during 1988-1990 (inclusive) and was prospective in nature. A note was made of the type of biopsy (one, two or three specimens) and the nature (and management) of complications in all patients. In addition, the nature of the aftercare of patients was recorded during the second part of the study. The clinicians caring for the patients made their own choices regarding the type of biopsy and the frequency of observations post-biopsy; the investigators did not influence these variables and were only responsible for recording the data.

Biopsies were carried out in the general medical and surgical wards of the hospital by interns after consent for the procedure was obtained from the patient. A platelet count greater than $100 \times 10^{9} / 1$ and a prothrombin index greater than $75 \%$ was considered a prerequisite for the performance of 
this procedure. After infiltration of the skin and subcutaneous tissue with $2 \%$ lignocaine, the biopsy was performed using a 'Tru-Cut' needle. Based upon their individual preferences, the doctors would take either one, two or three specimens; the additional specimens were obtained by introducing the biopsy needle through a single entry site and redirecting it by about $20^{\circ}$ to $30^{\circ}$ from the initial position. ${ }^{1}$ Thereafter, the nursing staff were requested to monitor the pulse rate and blood pressure. Some interns prescribed an opioid analgesic, usually pethidine, before the procedure. Patients were kept in hospital for at least 24 hours after biopsy.

\section{Results}

A total of 2,646 percutaneous biopsies were carried out, 1,525 in the first part of the study and 1,121 in the second part. A single specimen was obtained in 834 patients, two specimens in 983 patients and three in 829 patients. The morbidity and mortality rates associated with this procedure were $2.1 \%$ and $0.3 \%$, respectively. The nature and frequency of complications are shown in the Table I. Complications directly attributable to the procedure occurred in 24 patients who had one specimen, 20 who had two and 19 who had three specimens taken during the biopsy. Abdominal, thoracic or shouldertip pain was usually experienced soon after biopsy and responded to analgesics. Symptomatic hypotension (systolic blood pressure $<100 \mathrm{mmHg}$ ) was corrected by the administration of intravenous fluids, freeze-dried plasma or blood; none of the patients needed a laparotomy. The patient who developed biliary peritonitis required a laparotomy. A single specimen had been obtained from three of the eight patients who died, two specimens were taken from another patient and three specimens had been obtained from the other four patients. The final diagnosis in the eight patients who died were hepatocellular carcinoma $(n=5)$, Kaposi's sarcoma $(n=1)$, disseminated tuberculosis plus alcoholic hepatitis $(n=1)$ and pelvic and lung abscesses $(n=1)$. Intraperitoneal bleeding was the cause of death in all patients.

\section{Discussion}

Biopsy of the liver is a valuable method of detecting hepatic disease. However, important pathology can be overlooked when blind percutaneous needle biopsy of the liver is performed in the conventional manner because of the influence of sampling variability. ${ }^{1}$ The diagnostic yield of this procedure is improved when two or three consecutive biopsy specimens are obtained by introducing the biopsy needle through a single entry site and redirecting it by about $20^{\circ}$ to $30^{\circ}$ from the initial position. ${ }^{1}$

The present study in 2,646 patients indicates that this can be achieved without an increase in morbidity and mortality; patients in whom two or three specimens were taken (983 and 829 patients, respectively) did not have a higher incidence of fatalities, pain, symptomatic hypotension, or biliary peritonitis than those in whom a single specimen was obtained ( 834 patients). These findings are in agreement with those of an earlier investigation in which 344 patients required between two and 11 biopsies; an increased frequency of complications, all minor in nature, was observed after more than three consecutive biopsies were performed. ${ }^{2} \mathrm{~A}$ similar conclusion was reached by a group of workers who performed multiple liver biopsies under ultrasonographic guidance on 95 patients with carcinoid and endocrine pancreatic tumours. ${ }^{3}$ The only major complication in this study, intrahepatic haematoma, occurred in three patients who had required more than three biopsies. Thus it appears that the safety of both blind and imagingguided needle biopsy of the liver is adversely affected by taking more than three consecutive

Table I Morbidity and mortality following percutaneous liver biopsy when one, two or three specimens were taken

\begin{tabular}{|c|c|c|c|c|}
\hline & \multicolumn{3}{|c|}{ Number of patients } & \multirow[b]{2}{*}{ Total } \\
\hline & $\begin{array}{c}\text { One } \\
\text { specimen } \\
(\mathrm{n}=834)\end{array}$ & $\begin{array}{c}\text { Two } \\
\text { specimens } \\
(\mathrm{n}=983)\end{array}$ & $\begin{array}{c}\text { Three } \\
\text { specimens } \\
(\mathrm{n}=829)\end{array}$ & \\
\hline Pain & 12 & 12 & 7 & 31 \\
\hline \multicolumn{5}{|l|}{ Symptomatic } \\
\hline hypotension & 9 & 6 & 8 & 23 \\
\hline Biliary peritonitis & 0 & 1 & 0 & 1 \\
\hline Death & 3 & 1 & 4 & 8 \\
\hline Total & 24 & 20 & 19 & 63 \\
\hline
\end{tabular}


specimens.

Our data need to be viewed against the background that clinicians chose to take a single specimen in approximately one-third of patients requiring liver biopsy during the study period despite being aware of the influence of sampling variability on the diagnostic yield of blind liver biopsy (which could be improved by taking additional specimens during the procedure). Many stated that there were insufficient data on the safety of the procedure when more than one specimen was taken and were accordingly unable to justify the possible increased risk of complications that could accompany a possible increase in diagnostic yield. Also, our data on complications would probably have given clinicians more assurance had an increased diagnostic yield been demonstrated in the patients who had two or three specimens taken compared to one specimen. This was, unfortunately, not done because at the time that this study was being designed, the need for such an analysis was not foreseen; it was felt that a previous publication which contained our data and those of other workers, indicated conclusively that there was sampling variability in blind percutaneous needle biopsy of the liver and that pathology could be missed if only a single specimen was taken.

The morbidity and mortality rates associated with liver biopsy in this hospital are high. It could be argued that the prognosis in the majority of patients who died was extremely poor. Nonetheless, a critical evaluation of the factors that could have predisposed to complications was carried out. It emerged that in the majority of patients in this study, the after-care and monitoring were either inadequate or non-existent. During the last year of the study, post-biopsy monitoring was requested in only $171(45.6 \%)$ patients, done in only 130 $(34.7 \%)$ patients, and lasted less than 4 hours in 70 $(18.7 \%)$. In addition, of the patients who died, only one had been adequately monitored after liver biopsy; a gradual reduction in blood pressure had been detected, but no remedial measures were instituted until shortly before his death. In the other patients, the first blood pressure measurement was made when it was apparent that a complication had occurred. This important duty in our hospital is delegated to nurses, who are already overcommitted as a result of staff shortages and overcrowding of wards. In order to reduce the mortality associated with liver biopsy, the doctor who carries out a percutaneous biopsy in developing countries will need to assume responsibility for monitoring the patient, for at least the first 4 hours after the biopsy - the period during which most of the serious complications are likely to become manifest. ${ }^{3}$ This will ensure that resuscitation and other appropriate measures will be instituted timeously. Further, though less frequent, observa- tions should be made over the next 18 hours because some patients may show signs of complications during this period. ${ }^{4}$

It is generally accepted that successful needle biopsy of the liver without complications demands good technique. ${ }^{4}$ Accordingly, interns must always be adequately trained. ${ }^{5}$ They also should be counselled to be prepared for the possible development of bleeding in all patients who are subjected to biopsy, even those who have no contraindications or are not considered to be at 'high-risk' for bleeding because this complication develops when least expected and when, at the time of the biopsy, the risk seemed small. ${ }^{4}$

Blind percutaneous needle biopsy of the liver is an important and valuable method for detecting liver disease in developing countries, ${ }^{1,6,7}$ especially those in which facilities for hepatic imaging and peritoneoscopy are not widely available. It is evident, however, that important pathological abnormalities can be overlooked in patients with hepatic disease when liver biopsy is done in the conventional manner. ${ }^{1}$ Also, it should be recognized that even in the best of hands, liver biopsy is associated with complications. Therefore, a clinician has to weigh up the possible benefit against the possible risk of the procedure. Having decided that biopsy is necessary, the aim should be to obtain the maximum information without increasing the risk of complications. Accordingly, obtaining two spec imens during blind percutaneous needle biopsy of the liver would be a reasonable compromise between the standard method of taking a single specimen, which is inadequate, and obtaining three specimens, which may be viewed as excessive. A trained operator ${ }^{4}$ should perform the procedure ${ }^{5}$ and two specimens should be taken ${ }^{7}$ by redirecting the needle through a single entry site as this improves the diagnostic yield of the biopsy without increasing the risk of complications. Obtaining two specimens during blind percutaneous needle biopsy of the liver represents a reasonable compromise between the standard method of taking a single specimen, which is inadequate, and obtaining three specimens, which may be viewed as excessive. Greater awareness of the hazards of liver biopsy and their prevention is needed in these countries; good technique, careful monitoring, and prompt and aggressive resuscitation are essential if the mortality rate is to be reduced.

\section{Acknowledgements}

Dr I.G. Bhoora was the recipient of a South African Medical Research Council Post-Intern Scholarship. We thank Mr Woogen Reddy and Mr Jerome Swinney for their assistance during the study and Mrs Odette French for typing the manuscript. 


\section{References}

1. Maharaj, B., Maharaj, R.J., Leary, W.P. et al. Sampling variability and its influence on the diagnostic yield of percutaneous needle biopsy of the liver. Lancet 1986, i: 523-525.

2. Perrault, J., McGill, D.B., Ott, B. \& Taylor, W.F. Liver biopsy: complications in 1000 inpatients and outpatients. Gastroenterology 1978, 74: 103-106.

3. Andersson, T., Eriksson, B., Lindgren, P.G., Wilander, E. \& Oberg, K. Percutaneous ultrasonography - guided cutting biopsy from liver metastases of endocrine gastrointestinal tumors. Ann Surg 1987, 206: 728-732.

4. Sherlock, S. Diseases of the liver and biliary system. In: Diseases of the Liver and Biliary System. Blackwell Scientific Publications, Oxford, 1985, pp. 28-37.
5. Maharaj, B. \& Pillay, S. 'Tru-Cut' needle biopsy of the liver: importance of the correct technique. Postgrad Med J 1991, 67: 170-173.

6. Maharaj, B., Cooppan, R.M., Maharaj, R.J. et al. Causes of hepatomegaly at King Edward VIII Hospital. Durban. A prospective study of 240 black patients. $S$ Afr Med J 1986, 69: 183-184.

7. Maharaj, B., Leary, W.P. \& Pudifin, D.J. A prospective study of hepatic tuberculosis in 41 black patients. $Q J$ Med 1987, 63: 517-522. 